Methods In this cross-sectional study, 27 prisons across 16 provinces were selected using a stratified random sampling approach. Men aged $\geq 18$ years who spent at least 1 week in the prison and self-reported having had sex during last year were eligible for this analysis. Participants were asked about their current STI symptoms including penile discharge (PD) and genital ulcers (GU). Demographic variables, HIV/STIrelated knowledge, STI care seeking practices, HIV self-perceived risk, as well as history of substance use, incarceration, and sexual behaviours were collected using a face-to-face pilot-tested risk assessment questionnaire. HIV tests were completed using ELISA of dried blood spots. Correlates of having STI symptoms were identified using descriptive statistics and logistic regression models.

Results Out of 2610 male prisoner recruited (Mean age \pm SD: $35.7 \pm 0.19$ ), $7 \%$ reported symptoms for PD, UC, or both; $45 \%$ of whom had not sought STI care inside prisons. Having STI-related symptoms were positively associated with a history of injection drug use (adjusted odds ratio [AOR]: 2.1, 95\% CI, 1.4-3.1), condom accessibility inside prison (AOR: 1.7, 95\% CI , 1.1-2.8), self-perceived risk of HIV (AOR: 1.5, 95\% CI , 1.1-2.2), HIV-seropositivity (AOR: 3.3, 95\% CI , 1.3-10.6), while negatively associated with having sufficient STI-related knowledge (AOR: 0.6, 95\% CI, 0.4-0.8).

Conclusion STI symptoms are notable among prisoners in Iran with a higher prevalence among specific groups (i.e., those who inject drugs and live with HIV). Our findings call for revisiting current HIV/STI prevention policies across Iranian prisons to help improve prisoners' HIV/STI knowledge and encourage their HIV/STI preventive practices.

Disclosure No significant relationships.

\section{P809 NEGOTIATING SAFER SEXUAL RELATIONS WITH HUSBAND AND ASSOCIATED STI/HIV VULNERABILITIES AMONG MARRIED WOMEN IN INDIA}

Deepanjali Vishwakarma*, Santosh Sharma. International Institute for Population Sciences, Population Studies, Mumbai, India

\subsection{6/sextrans-2019-sti.858}

Background In India, sexually transmitted infections (STIs) and HIV, take an enormous toll on women's sexual/reproductive health, yet preventive programmes are lacking as married women's risks are frequently underestimated. Husbands continue to be the greatest source of STIs including HIV to their wives. Being able to negotiate safe sex is critical to the prevention of HIV/AIDS and other STIs. Therefore, the present study has mainly focused to analyze the women's attitudes toward safer-sex negotiation if husband has a STI and associated risk of STI/HIV among married women in India.

Methods National Family Health Survey (NFHS-4, 2015-16) collected information from a nationally representative sample of 121,118 women age 15-49 years has been analyzed by using descriptive and multivariate techniques. Women were asked that a wife is justified in refusing to have sexual intercourse with her husband if he has a STI.

Results Overall $78 \%$ of women agree that a wife is justified in refusing to have sex with her husband if he has a STI. A higher proportion of women who were agreed that a wife is justified to negotiate sex were from 25-29 years of age, residing in urban area, those who were higher educated and belongs to highest wealth quintile. Results revealed that the likelihood of a woman holding this belief increased with her autonomy, as measured by participation in household decision making and rejection of wife beating $(\mathrm{p}<0.001)$. Women who were away from home for one or more month were significantly less likely to agree. Other significant predictors were knowledge/awareness of STIs (OR-1.13 p $<0.01)$. Those women who agreed towards negotiating sex were significantly less likely to have a STI.

Conclusion Our findings suggest that sexual health education programmes may be more effective if they include strategies to address social norms and cultural practices that limit women's autonomy in society.

Disclosure No significant relationships.

\section{P810 HIVISTI SERVICE COVERAGE AMONG KEY POPULATION IN NIGERIA - LESSONS FROM SIZE ESTIMATION STUDY IN ABIA AND TARABA STATES}

${ }^{1}$ Greg Ashefor*, ${ }^{1}$ Adaoha Anosike, ${ }^{1}$ Idoteyin Ezirim, ${ }^{1}$ Olutosin Adebanjo, ${ }^{2}$ Chukumebuka Ejeckam, ${ }^{3}$ Kalada Green. ${ }^{1}$ National Agency for the Control of AIDS, Research Monitoring and Evaluation, Abuja, Nigeria; ' University of Manitoba, Abuja, Nigeria; ${ }^{3}$ Centre for Global Public Health - Nigeria, Abuja, Nigeria

\subsection{6/sextrans-2019-sti.859}

Background Mode of transmission study revealed that 38\% of HIV new-infections in Nigeria are attributable to KPs. Abia and Taraba are two states in Nigeria with an HIV prevalence of $3.9 \%$ and $5.1 \%$ respectively. To ensure KPs receive interventions, estimation study was conducted in both states to provide insight on KP size, distribution and HIV/STI service coverage.

Methods Three KP groups (FSW, PWID, MSM) were mapped in Abia and Taraba. Programmatic mapping was employed which involved two sequential data collection steps known as level one [L1] and level two [L2]. During L1, data was collected from key informants (KIs) on the geographic locations/ spots where KPs congregate, the characteristics of the spots, estimate of KPs found there and HIV/STI service availability. During L2, KI interviews were conducted at spots identified in L1. In L2 interviews primary KIs (FSWs, IDUs, MSM,) validated information collected during L1.

Results 1,136 spots (679 FSW spots, 103 MSM spots, 354 PWID spots) were identified in Abia while Taraba had 574 spots (346 FSW spots, 98 MSM spots, 130 PWID spots). The total KP estimate in Abia is 13,527 while Taraba has 6,246. In Abia, condom and HIV testing were only available in $4.4 \%$ and $1.6 \%$ FSW spots respectively. Also condom and HIV testing were only available in $2.8 \%$ and $1.1 \%$ PWIDs spots respectively. Both services weren't available at MSM spots while all KPs spots had no STI services. In Taraba, condom and HIV testing were only available in $0.6 \%$ and $1.3 \%$ FSW spots respectively. Also condom and HIV testing were only available in $4.2 \%$ and $3.4 \%$ PWIDs spots respectively. Both services weren't available at MSM spots. 0.3\% FSW spots had STI service but other KP spots had no STI services.

Conclusion From this study, Nigeria needs to scale up targeted HIV/STI services for KPs in Abia and Taraba states.

Disclosure No significant relationships. 